\title{
Identification of the dominant non-neutralizing epitopes in the haemagglutinin of H7N9 avian influenza virus
}

\author{
Zenglei $\mathrm{Hu}^{1}$, Jiangyan Zhao ${ }^{2}$, Lei $\mathrm{Shi}^{1}$, Jiao $\mathrm{Hu}^{1}$, Xiaoquan Wang ${ }^{1}$, Xiaowen $\mathrm{Liu}^{2}$, Shunlin \\ $\mathrm{Hu}^{1}$, Min $\mathrm{Gu}^{1}$, and Xiufan $\mathrm{Liu}^{2}$ \\ ${ }^{1}$ Affiliation not available \\ ${ }^{2}$ Yangzhou University
}

October 5, 2020

\begin{abstract}
H7N9 avian influenza vaccines induce high levels of non-neutralizing (nonNeu) antibodies against the haemagglutinin (HA). However, the antigenic epitopes underlying this particular antibody response are still undefined. In this study, a panel of 15 monoclonal antibodies (mAbs) against the HA protein of H7N9 virus was generated and 14 of them had no hemagglutination inhibition and virus neutralizing activities. Four antigenic epitopes, including one linear and three conformational epitopes, in HA were identified using peptide-based enzyme-linked immunosorbent assay and biopanning of phage display random peptide library. More importantly, two mAbs (4B7 4D5 and 1B10 1D1) strongly inhibited HA-binding of chicken nonNeu antisera against viral-vectored H7N9 vaccine, whereas lower inhibition was observed for chicken neutralizing antisera. In contrast, there was weak competition between the $\mathrm{mAb}$ and murine nonNeu antisera against inactivated H7N9 antigen. The epitopes targeted by these two mAbs were defined as the immunodominant epitopes underpinning the elicitation of nonNeu antibodies by viral-vectored H7N9 vaccine. Additionally, the identified stalk epitopes were conserved among the H1-H17 subtypes and the stalk-reactive mAbs exhibited cross-reactivity with different subtypes. In conclusion, four novel nonNeu epitopes in H7N9 HA were identified, and two dominant epitopes underlying the induction of nonNeu antibodies by viral-vectored H7N9 vaccine were identified. Our results add new knowledge to the molecular basis for antibody immunity against H7N9 vaccines and provide useful implications for vaccine design and modification.
\end{abstract}

\section{Identification of the dominant non-neutralizing epitopes in the haemagglutinin of H7N9 avian influenza virus}

Zenglei $\mathrm{Hu}^{1,2,3,4+}$, Jiangyan Zhao ${ }^{1+}$, Lei $\mathrm{Shi}^{1}$, Jiao $\mathrm{Hu}^{1,2,3}$, Xiaoquan Wang1, 2, 3, Xiaowen Liu ${ }^{1,2,3}$, Shunlin $\mathrm{Hu}^{1,2,3}$, Min $\mathrm{Gu}^{1,2,3}$, Xiufan Liu ${ }^{*}$

${ }^{1}$ Animal Infectious Disease Laboratory, School of Veterinary Medicine, Yangzhou University, Yangzhou, China

2 Jiangsu Co-innovation Center for Prevention and Control of Important Animal Infectious Diseases and Zoonoses, Yangzhou University, Yangzhou, China

${ }^{3}$ Jiangsu Key Laboratory of Zoonosis, Yangzhou University, Yangzhou, China

${ }^{4}$ Joint International Research Laboratory of Agriculture and Agri-Product Safety, The Ministry of Education of China, Yangzhou University, Yangzhou, China

+ Equal contribution.

Running title: dominant non-neutralizing epitopes in H7N9 HA 
* Correspondence:

Xiufan Liu, E-mail: xfliu@yzu.edu.cn

Mail address: No. 48, East Wenhui Rd, Yangzhou, Jiangsu Province, China, 225009

Abstract

H7N9 avian influenza vaccines induce high levels of non-neutralizing (nonNeu) antibodies against the haemagglutinin (HA). However, the antigenic epitopes underlying this particular antibody response are still undefined. In this study, a panel of 15 monoclonal antibodies (mAbs) against the HA protein of H7N9 virus was generated and 14 of them had no hemagglutination inhibition and virus neutralizing activities. Four antigenic epitopes, including one linear and three conformational epitopes, in HA were identified using peptide-based enzyme-linked immunosorbent assay and biopanning of phage display random peptide library. More importantly, two mAbs (4B7 4D5 and 1B10 1D1) strongly inhibited HA-binding of chicken nonNeu antisera against viral-vectored H7N9 vaccine, whereas lower inhibition was observed for chicken neutralizing antisera. In contrast, there was weak competition between the $\mathrm{mAb}$ and murine nonNeu antisera against inactivated H7N9 antigen. The epitopes targeted by these two mAbs were defined as the immunodominant epitopes underpinning the elicitation of nonNeu antibodies by viral-vectored H7N9 vaccine. Additionally, the identified stalk epitopes were conserved among the H1-H17 subtypes and the stalk-reactive mAbs exhibited cross-reactivity with different subtypes. In conclusion, four novel nonNeu epitopes in H7N9 HA were identified, and two dominant epitopes underlying the induction of nonNeu antibodies by viral-vectored H7N9 vaccine were identified. Our results add new knowledge to the molecular basis for antibody immunity against H7N9 vaccines and provide useful implications for vaccine design and modification.

Keywords: H7N9 avian influenza virus; haemagglutinin; non-neutralizing antibody; epitope; vaccine; antibody immunity

Introduction

Avian influenza A (H7N9) virus has emerged as a public health concern in China (Gao et al., 2013). A total of five waves of the H7N9 epidemic have hit China from 2013 to 2017, leading to high mortalities of humans (Wang et al., 2017; Zhang et al., 2017). Highly pathogenic (HP) avian influenza H7N9 viruses have emerged during the fifth wave and pose a severe challenge for both poultry industry and public health (Jiang et al., 2019; Ma, Yang, \& Fang, 2018). Human cases of H7N9 infection and the isolation rate of H7N9 viruses in poultry dropped sharply since October 2017 due to the implementation of vaccination campaign nationwide (Shi et al., 2018). However, according to a latest epidemiological study, H7N9 viruses still have the potential to cause the next pandemic because they have well adapted in ducks (Shi et al., 2018).

After the emergence of H7N9 avian influenza, many vaccine candidates have been generated using different technology platforms (Isakova-Sivak \& Rudenko, 2017). Animal experiments and human clinical trials revealed that $\mathrm{H} 7 \mathrm{~N} 9$ vaccines are generally immunogenic and efficacious and the antibody immunity induced by H7N9 vaccines is characterized with low neutralizing antibody but high levels of non-neutralizing (nonNeu) antibody (Bart et al., 2014; Jackson et al., 2015; Kamal et al., 2017; Krammer \& Cox, 2013). These findings suggest that novel antigenic epitopes outside the classical neutralizing epitopes may be immunodominant in the haemagglutinin (HA). Currently, neutralizing monoclonal antibodies (mAbs) against H7N9 viruses were mainly used for epitope mapping of the HA protein using the traditional method of generating immune escape mutants (Sun et al., 2018; Thornburg et al., 2016; Yang et al., 2020). However, antigenic epitopes in HA underlying the induction of nonNeu antibodies, especially the dominant epitopes in the context of polyclonal antibody response to H7N9 vaccines, remain undefined.

In this study, a panel of 15 murine mAbs against the HA protein of an HP H7N9 strain (A/chicken/Guangdong/GD15/2016, GD15) was generated using hybridoma method. Hemaglutination inhibition (HI), virus neutralizing (VN), HA-binding and reactivity with H7N9 virus of the mAbs were determined. The epitopes recognized by these mAbs were mapped using peptide-based enzyme-linked immunosorbent assay (ELISA) and/or biopanning of phage display random peptide library. Competition 
ELISA between the mAbs and H7N9 antisera was performed to identify the immunodominant epitopes in HA. In addition, sequence variation of the identified stalk epitopes and cross-reactivity of the stalk-specific mAbs with different subtypes were analyzed. Four new nonNeu epitopes in HA of H7N9 virus were identified and two of them were defined as the immunodominant epitopes. Our findings present new information for understanding the immunogenicity of $\mathrm{H} 7 \mathrm{~N} 9$ vaccines and provide useful information for vaccine development.

1. Materials and methods

2. Viruses, cells and chicken antisera

H7N9 GD15 strain and its attenuated derivate rGD15 were described previously (Hu et al., 2019; Liu et al., 2018). The viruses were propagated in 10-day-old embryonated chicken eggs. Insect sf9 cells were cultured in EX-CELL ${ }^{\circledR} 420$ medium (Sigma, St. Louis, MO, USA) supplemented with $5 \%$ fetal calf serum (FCS) at $27^{\circ} \mathrm{C}$. Chicken embryo fibroblasts (CEFs) were grown in M199 medium supplemented with $4 \%$ FCS at $37^{\circ} \mathrm{C}, 5 \% \mathrm{CO} 2$. Madin-Darby canine kidney (MDCK) cells and the myeloma cell line SP2/0 were cultured in Dulbecco's Modified Eagle Medium supplemented with $10 \%$ FCS $37^{\circ} \mathrm{C}, 5 \%$ CO2. The antisera from chickens immunized with Newcastle disease virus (NDV)-vectored or inactivated H7N9 vaccine were obtained from previous studies (Hu et al., 2019; Shi et al., 2019). Of note, the NDV-vectored H7N9 vaccine sera have no virus neutralizing activity and the inactivated H7N9 vaccine sera have high neutralizing titers.

Preparation of nonNeu antisera against H7N9 in mice

To prepare anti-H7N9 sera in mice, rGD15 was inactivated with formalin and then purified using ultracentrifugation at 30,000 rpm for $2 \mathrm{~h}$. The HA content in the purified virus was quantified using sodium dodecyl sulfate polyacrylamide gel electrophoresis (SDS-PAGE) as described elsewhere (Wong et al., 2014). Three female 8-wk-old BALB/C mice were subcutaneously (s.c.) immunized with $0.2 \mathrm{~mL}$ of the H7N9 antigen (containing $3 \mu \mathrm{g}$ of the HA protein) in Freund's complete adjuvant (Sigma) and boosted with the antigen mixed with Freund's incomplete adjuvant (Sigma) at the same dose after two weeks. Another three mice were immunized twice s.c. with non-adjuvanted H7N9 antigen using the same procedure. The sera were collected at week 2 after booster to measure HI, VN and IgG antibody titers.

Generation of the mAbs against the H7N9 HA protein

The HA protein was expressed in baculovirus expression system (BES). The ectodomain (1-1,587 nt) of the HA gene of rGD15 in fusion with $6 \times$ His tag at the C-terminal was amplified and cloned into the baculovirus transfer vector $\mathrm{pVL} 1393$. The recombinant baculovirus was generated by co-transfecting the transfer plasmid together with the linearized Sapphire ${ }^{\mathrm{TM}}$ baculovirus DNA (Allele Biotechnology, San Diego, CA, USA) into sf9 cells. The recombinant baculovirus was plaque-purified and then propagated in sf9 cells. Expression of the HA protein was determined in the cells inoculated with the recombinant baculovirus using indirect immunofluorescence assay (IFA). $500 \mathrm{~mL}$ of sf9 suspension culture was inoculated with the recombinant baculovirus to produce the recombinant HA protein, which was purified by Ni-NTA column and identified using SDS-PAGE and Western blot.

To generate HA-specific mAbs, eight 6-wk-old BALB/c mice were s.c. immunized with $50 \mu \mathrm{g}$ of the purified HA protein with Freund's complete adjuvant. The mice were boosted with $50 \mu \mathrm{g}$ of the HA protein with Freund's incomplete adjuvant for three times at an interval of two weeks. Seven days after the final boost, antibody titers were measured and spleen cells were collected for fusion with myeloma cells SP2/0. At day 7 after cell fusion, the hybridoma supernatants were screened using indirect ELISA as described below. The positive clones were then sub-cloned using limiting dilution and characterized by ELISA. The hybridoma supernatants from the positive clones were used for isotyping, antibody characterization, peptide-based and competition ELISA. BALB/c mice were intraperitoneally injected with the hybridoma cells to produce ascites, which were used to purify the mAbs through protein $\mathrm{G}$ affinity chromatography (GE healthcare, Piscataway, NJ, USA).

Biological characterization of the mAbs

HI, VN and HA-binding activities of the mAbs were measured. HI titers against rGD15 were measured 
according to the standard protocol. Microneutralization assay was conducted to determine VN activity of the antibodies. In brief, the antibodies were 2-fold serially diluted and then incubated with $10050 \%$ tissue culture infectious dose $\left(\right.$ TCID $\left._{50}\right)$ of HP H7N9 GD15 strain at $37^{\circ} \mathrm{C}$ for $1 \mathrm{~h}$. rGD15 antiserum was used as the positive control, which was heat-inactivated at $56^{\circ} \mathrm{C}$ for $0.5 \mathrm{~h}$ prior to dilution. The mixture was then transferred to CEFs and cultured for $72 \mathrm{~h}$. The culture supernatants were then collected for hemagglutination (HA) test to determine virus infection. The highest dilution of the antibodies that can totally inhibit virus replication was considered as the $\mathrm{VN}$ titer.

ELISA was conducted to assess binding of the mAbs to the HA protein. Briefly, microtiter plates were coated overnight with the HA protein $(0.25 \mu \mathrm{g} / \mathrm{mL})$ in $100 \mu \mathrm{L}$ of carbonate-bicarbonate buffer $(0.05 \mathrm{M}$ $\mathrm{Na}_{2} \mathrm{CO}_{3} / \mathrm{NaHCO}_{3}, \mathrm{pH} 9.6$ ) at $4^{\circ} \mathrm{C}$. The plates were washed three times with PBST (PBS containing 0.05 $\%$ Tween-20) and then blocked with $100 \mu \mathrm{L}$ of $5 \mathrm{mg} / \mathrm{mL}$ BSA in PBST at $37^{\circ} \mathrm{C}$ for $1 \mathrm{~h}$. After the blocking step, the plates were washed three times with PBST and then incubated with the hybridoma supernatants at $37^{\circ} \mathrm{C}$ for $1 \mathrm{~h}(100 \mu \mathrm{L} /$ well $)$. After washing, $100 \mu \mathrm{L}$ of the secondary anti-mouse IgG HRP antibody at a dilution of 1:5,000 was added and incubated for another $1 \mathrm{~h}$ at $37^{\circ} \mathrm{C}$. The plates were washed with PBST for three times and then developed with $100 \mu \mathrm{L}$ of TMB substrate at room temperature for $10 \mathrm{~min} .50 \mu \mathrm{L}$ of $3 \mathrm{M} \mathrm{HCl}$ was added to stop the reaction and the plates were read under $450 \mathrm{~nm}$.

In addition, reactivity of the mAbs with H7N9 virus was assesses in virus-infected cells using IFA. In brief, $5 \times 10^{5}$ MDCK cells were seeded in 6-well culture plates and inoculated with GD15 at a multiplicity of infection (MOI) of 0.1. At $30 \mathrm{~h}$ post-inoculation, the cells were fixed with cold methanol, incubated with the indicated anti-H7N9 mAbs at different dilutions and then with goat anti-mouse IgG Alexa 488 antibody. Fluorescence was monitored under a microscope.

\section{Peptide-based ELISA}

Potential B cell epitopes in $\mathrm{H} 7 \mathrm{HA}$ were predicted using a web-based prediction tools BepiPrep (http://www.cbs.dtu.dk/services/BepiPred-1.0/). The predicted B cell epitopes were synthesized and an additional cysteine residue was added at the $\mathrm{N}$ - or C-terminal for bovine serum albumin (BSA) conjugation. Eleven peptides representing potential B cell epitopes in the stalk as reported previously were also included in this study (Hu et al., 2020). Peptide-based ELISA was performed to map the epitopes recognized by the mAbs. The procedure of peptide-ELISA was similar to indirect ELISA as described above except for that the plates were coated with the synthesized peptides $(1 \mu \mathrm{g} /$ well $)$. The screened peptides were further truncated for fine mapping of the linear epitopes of the antibodies. Moreover, peptide-based ELISA was also performed to characterize reactivity of H7N9 antisera with the potential B cell epitopes in HA.

\section{Competition ELISA}

To identify the dominant epitopes in HA targeted by nonNeu antibodies against H7N9 virus, the hybridoma supernatants were first screened for their competition capacity with chicken nonNeu antisera against NDVH7N9 vaccine through competition ELISA. Briefly, microtiter plates were coated with the HA protein $(0.25$ $\mu \mathrm{g} / \mathrm{mL})$ at $4^{\circ} \mathrm{C}$ overnight. After washing, the plates were blocked with $100 \mu \mathrm{L}$ of PBST containing $5 \mathrm{mg} / \mathrm{mL}$ BSA at $37^{\circ} \mathrm{C}$ for $1 \mathrm{~h}$. The plates were washed three times with PBST and then incubated with the hybridoma supernatants at $37^{\circ} \mathrm{C}$ for $1 \mathrm{~h}(100 \mu \mathrm{L} /$ well). The culture supernatant collected from SP2/0 cells was used as the negative control. After washing, $100 \mu \mathrm{L}$ of chicken antiserum (1:200) was added and incubated at $37^{\circ} \mathrm{C}$ for $1 \mathrm{~h}$. The anti-chicken IgY HRP antibody (1:2,000) was added and incubated for another $1 \mathrm{~h}$ at $37^{\circ} \mathrm{C}$. The plates were then developed with $100 \mu \mathrm{L}$ of TMB substrate at room temperature for $10 \mathrm{~min}$. Then $50 \mu \mathrm{L}$ of $3 \mathrm{M} \mathrm{HCl}$ was added to stop the reaction. The OD values with and without inhibitor (hybridoma supernatants) were measured under $450 \mathrm{~nm}$, which were used to calculate the percentage of inhibition (PI) according to the following formula: PI $=(1-\mathrm{OD}$ with inhibitor $/$ OD without inhibitor $) \times 100$.

Based on the screening data, two purified mAbs (4B7 4D5 and 1B10 1D1) were selected for competition ELISA with chicken nonNeu and neutralizing antisera. The mAbs were 10 -fold serially diluted and used as inhibitor individually or combined in competition ELISA. Moreover, 4B7 4D5 was conjugated with HRP according to the manufacturer's instruction (Proteintech, Wuhan, China) and used in competition ELISA 
between the mAb and mouse antisera. Serially diluted mouse anti-H7N9 sera (as the inhibitor) were incubated with the HA protein and then the HRP-conjugated 4B7 4D5 was added. The sera collected from the nonimmunized mice were included as the negative control. PI was calculated as described above.

Solution-phase panning of phage display random peptide library

The Ph.D.-12 phage display peptide library (NEB, Ipswich, MA, USA) was used to map the epitopes of the indicated mAbs. In brief, $50 \mu \mathrm{L}$ of Protein A agarose was suspended in $1 \mathrm{~mL}$ of TBS $(50 \mathrm{mM}$ Tris- $\mathrm{HCl}$, $150 \mathrm{mM} \mathrm{NaCl}$ ) containing $0.1 \%$ Tween-20 (TBST). The resin was pelleted by low-speed centrifugation for $30 \mathrm{~s}$ and the supernatant was discarded. The agarose was suspended in $1 \mathrm{~mL}$ of blocking buffer $(5 \mathrm{mg} / \mathrm{mL}$ $\mathrm{BSA}$ in $0.1 \mathrm{M}$ NaHCO3) and incubated for $1 \mathrm{~h}$ at $4^{\circ} \mathrm{C}$. Meanwhile, $1 \times 10^{11}$ plaque forming unit of the phages was mixed with $0.3 \mu \mathrm{g}$ of the antibodies and incubated at room temperature (RT) for $20 \mathrm{~min}$. The phage-antibody mixture was transferred into the agarose and incubated at RT for $15 \mathrm{~min}$. Next, the resin was spin-down and washed with TBST for 10 times to remove unbound phages. The agarose was pelleted, suspended in $0.2 \mathrm{M}$ glycine elution buffer $(0.2 \mathrm{M}$ Glycine-HCl, $\mathrm{pH} 2.2,1 \mathrm{mg} / \mathrm{mL}$ BSA) and incubated at RT for $10 \mathrm{~min}$ to elute the bound phages. The supernatant was then transferred to a new tube and neutralized with $150 \mu \mathrm{L}$ of $1 \mathrm{M}$ Tris- $\mathrm{HCl}$. A small aliquot of the eluate was used for phage titration and the remaining eluate was amplified in $20 \mathrm{~mL}$ of E.coli ER2738 strain culture. After three successive rounds of panning, 10 individual phage clones were randomly picked up and amplified for DNA sequencing. The DNA inserts were sequenced using the -96 gIII sequencing primer: 5'-CCCTCATAGTTAGCGTAACG-3' according to the manufacturer's instructions. The amino acid sequences of these dodecapeptides deduced from the inserted nucleotide sequences were aligned to find the consensus sequence using BioEdit software. The mimotopes were then identified by alignment of the 12-peptide sequences with the amino acid sequence of HA.

Conformational analysis of the epitopes in HA and cross-reactivity of the stalk-specific mAbs

Localization of the four identified epitopes in the HA protein was analyzed using structure-modeling. Since the epitopes in the conserved stalk region was screened, variation of these epitopes among the H1-H17 subtypes was analyzed using BioEdit and illustrated using the Weblogo. Additionally, cross-reactivity of the stalk-specific mAbs against the representative subtypes from group 1 and 2 was evaluated. The HA proteins of the H1N1, H2N2, H5N1 and H9N2 subtypes belonging to group 1 and the H7N7, H7N9 and H15N8 subtypes belonging to group 2 (SinoBiological, Beijing, China) were used. The procedure of ELISA was as described above.

1. Results

2. Production of the recombinant HA protein in BES

The ectodomain of H7N9 HA was expressed in BES and used as the immunogen to generate HA-specific mAbs. The recombinant baculovirus was generated and expression of the HA protein was detected in sf9 cells inoculated with the virus using IFA (Figure 1a). $500 \mathrm{~mL}$ of sf9 suspension cell culture was inoculated with the recombinant baculovirus for large scale production of the HA protein. The HA protein with His-tag was purified by Ni-NTA column and the elution buffers containing $60 \mathrm{mM}$ imidazole resulted in the highest yield of the HA protein with the molecular weight of $\sim 66 \mathrm{kDa}$ (Figure $1 \mathrm{~b})$. In addition, the monomer $\left({ }^{\sim} 66\right.$ $\mathrm{kDa})$, dimer $(\sim 130 \mathrm{kDa})$ and trimer $(\sim 200 \mathrm{kDa})$ of the HA protein were detected using Western blot under non-reducing conditions (Figure 1c). These results showed that the HA protein of H7N9 virus was expressed in the form of monomer, dimer and trimer in baculovirus expression system.

Preparation of antisera against H7N9 in mice

Mice were immunized with the adjuvanted or non-adjuvanted inactivated H7N9 antigen to prepare antisera. As shown in Table 1, high HI antibody titers were detected in two out of three mice immunized with the adjuvanted H7N9 antigen, whereas the non-adjuvanted antigen failed to induce detectable HI titers in mice. Similarly, the non-adjuvanted H7N9 antigen elicited very low VN titers (1:40) in mice, whereas inclusion of the adjuvant significantly increased VN titers (mean titer 1:1066) against H7N9 virus. In addition, although the non-adjuvanted H7N9 antigen elicited low HI and VN titers, high levels of IgG antibody were induced, 
but the magnitude of IgG antibody response was lower than that for the adjuvanted antigen. These results suggested that the non-adjuvanted inactivated H7N9 antigen induced low HI/VN titers but high levels of IgG antibody in mice. Thus, the antisera obtained from the mice immunized with the non-adjuvanted H7N9 antigen were considered as nonNeu sera.

Generation and characterization of the mAbs

A panel of $15 \mathrm{mAbs}$ was generated from the mice immunized with the HA protein that reacted positively with the immunogen. The hybridoma supernatants were first used for antibody characterization. Interestingly, all the mAbs had strong binding activity with the HA protein, whereas they all had negative HI activity (Table 2). In addition, all the antibodies showed no virus neutralizing activity against H7N9 virus in CEFs, except for 2B11 1F3 exhibiting a low neutralizing activity (VN titer 1:40) (Table 2). Thus, a panel of HA-specific mAbs was generated and the overwhelming majority of these mAbs are nonNeu antibodies.

Epitope mapping of the mAbs

Peptide-based ELISA was first performed to identify the epitopes recognized by the nonNeu mAbs. Seven peptides predicted in this study, together with 12 previously-reported peptides (Hu et al., 2020), representing the potential epitopes basically encompassing the entire HA protein were synthesized (Supplemental Table 1). Peptide-based ELISA showed that four antibodies in total reacted positively with the peptides (Figure 2a-d). Among them, 1A1 1D7, 1A12 2A4 and 2E3 1D8 showed high binding with both the peptide 12 and 13 because these two peptides have an overlap (373-TAADYKSTQSA-383) (Figure 2a-c). The mAb 5D3 1B5 had strong reactivity with the peptide 19 (Figure 2d). The remaining 11 antibodies did not react with all the tested peptides (data not shown).

To further perform fine mapping of the epitopes, the screened peptides were truncated into shorter overlapping peptides. The overlap motif between the peptide 12 and 13 was truncated into three short peptides (Figure 2e). The results demonstrated that 1A1 1D7, 1A12 2A4 and 2E3 1D8 exhibited high reactivity with the peptide 12/13-1, comparable to that with the peptide 13. In contrast, no reaction of these three antibodies with the other two truncated peptides was seen (Figure 2g-i). Therefore, a short motif "373-TAA-375" in the stalk region of HA was identified as the epitope for these three antibodies. The peptide 19 recognized by 5D3 1B5 was truncated into five overlapping short peptides (Figure 2f). Unexpectedly, 5D3 1B5 did not react with the truncated peptides derived from the peptide 19, indicating that this antibody may target conformational epitopes (Figure 2j). Therefore, biopanning of phage display library was conducted to screen the mimotope targeted by 5D3 1B5. Ten mAb-reactive phage clones were screened and amplified for DNA sequencing. Alignment of the peptide insert sequences resulted in a consensus sequence (AWQYSLTLPDVL) and three amino acids in this motif matched to residues W431, Y433 and L437 in the HA stalk. 431W-433Y437L was identified as the core sequence for the epitope recognized by 5D3 1B5 (Table 3) and this motif located in the peptide 19 ranging from residues 428 to 452 . Therefore, two new epitopes in the HA stalk targeted by nonNeu mAbs were identified using peptide-based ELISA or/and phage library panning.

Reactivity of the mAbs with H7N9 virus

The mAbs showed high binding activity with the HA protein as determined by ELISA. Reactivity of the indicated mAbs with H7N9 virus was also investigated in virus-infected MDCK cells using IFA. We found that all the tested antibodies, 1A1 1D7, 1B10 1D1, 5D3 1B5 and 4B7 4D5, that were 1:500 diluted recognized GD15 virus in the cells, and no fluorescence was detected in the mock cells (Figure 3). Various concentrations of the mAbs were tested in IFA and the highest dilution of 1A1 1D7 reacted with the virus was 1:2,000, whereas the other three still reacted with the virus at 1:8,000 dilution (data not shown), suggesting the stronger reactivity of these antibodies. These data demonstrated that anti-H7N9 mAbs can recognize the H7N9 antigen expressed in the process of virus infection.

Identification of the dominant nonNeu epitopes in the HA protein

To identify the immunodominant epitopes underlying the induction of nonNeu antibodies by H7N9 vaccines, reactivity of nonNeu H7N9 antisera obtained from immunized chickens and mice with the peptides was 
first determined. Interestingly, both the chicken and mouse nonNeu H7N9 sera did not react with all the 19 peptides, while exhibiting high reactivity with the HA protein (Figure 4a, b). Next, the hybridoma supernatants without neutralizing activity were first screened for their inhibitory effect on the NDV-H7N9 antisera using competition ELISA. HA-binding of the sera decreased by around 50 and $20 \%$ in the presence of 4B7 4D5 and 1B10 1D1, respectively (Figure 4c). Moreover, the purified 4B7 4D5 significantly inhibited HAbinding of the NDV-H7N9 sera even at a high dilution (10,000-fold), whereas this mAb had a lower inhibitory effect on the rGD15 sera ( $20 \%$ PI observed for 100-fold diluted mAb) (Figure 4d, e, g, h). In addition, 1B10 1D1 also exhibited a potent inhibitory effect on the NDV-H7N9 sera and the magnitude of inhibition was significantly lower compared to that for 4B7 4D5 (Figure 4d, g). A weak inhibition against the rGD15 sera was also observed for 1B10 1D1 (Figure 4e, h). It is noted that prior incubation of 1B10 1D1 resulted in a significantly lower inhibition for the NDV-H7N9 sera compared to 4B7 4D5 and additional inclusion of 4B7 4D5 markedly enhanced the inhibitory impact exerted by 1B10 1D1 (Figure 4d, g). Similarly, the presence of 4B7 4D5 plus 1B10 1D1 led to a significant increase of inhibition for the rGD15 sera compared to 1B10 1D1 alone (Figure 4e, h). However, a different profile of inhibition was observed for the mouse antisera. Both nonNeu and neutralizing mouse antisera had a low inhibition for HA-binding of 4B7 4D5 and no significant difference was observed (Figure 4f, i). Taken together, these results suggested that: 1) the conformational epitopes, instead of the linear epitopes, may be mainly targeted by H7N9 nonNeu antibodies; 2) the epitopes recognized by 4B7 4D5 and 1B10 1D1 were immunodominant in the HA protein; 3) different dominant epitopes may be targeted by nonNeu antibodies against viral-vectored and inactivated H7N9 vaccines.

Epitope mapping of the mAbs by phage peptide display library panning

The epitopes recognized by 4B7 4D5 and 1B10 1D1 were dominant in HA, but these two antibodies did not react with the linear peptides. Therefore, panning of 12-mer phage peptide display library was carried out to identify the mimotopes recognized by these antibodies. After three successive rounds of panning, ten positive phage clones for each antibody were screened and amplified for DNA sequencing. Sequence alignment of the inserts identified a consensus sequence SNTALPSKFYGY for 4B7 4D5 and four amino acids in this motif matched to residues N351, L356, Y361 and G362 in the HA stalk (Table 3). Additionally, the mimotope QxLSGT was identified for 1B10 1D1 by sequence alignment and three amino acids matched to residues Q220, S225 and G227 in the HA head (Table 3). Therefore, two immunodominant epitopes underlying the induction of nonNeu antibodies were identified in the HA protein of H7N9 virus.

Conformational analysis of the epitopes in HA and cross-reactivity of the stalk-specific mAbs

The epitope targeted by 1B10 1D1 locates in the 220-loop domain in the HA head, and the epitopes recognized by the stalk-reactive mAbs localize in the $\mathrm{C}$ helix (5D3 1B5) and A helix domain (4B7 4D5 and 1A1 1D7) in the HA stalk (Figure 5a). Sequence variation analysis demonstrated that the "373-TAA-375" motif targeted by 1A1 1D7 was conserved among different subtypes (Figure 5b). 373-T was detected in the H4, H14, H7 and H15 subtypes within group 2 instead of any subtypes within group 1, whereas 374-AA-375 was highly conserved among the H1-H17 subtypes. In addition, three amino acids 431W-433Y-451D targeted by 5D3 $1 \mathrm{~B} 5$ and two amino acids $361 \mathrm{Y}-362 \mathrm{G}$ recognized by $4 \mathrm{~B} 74 \mathrm{D} 5$ were highly conserved among the H1-H17 subtypes (Figure 5b). High variation of the three residues targeted by 1B10 1D1 was found because they locate in the HA head (Figure 5b). Cross-reactivity analysis showed that 5D3 1B5 had a cross-group reactivity with the HAs of the H1N1, H5N1 and H9N2 subtypes (group 1) and the H7N7, H7N9 and H15N8 subtypes (group 2) (Figure 5c). However, 1A1 1D7 only reacted positively with the HA proteins from group 2 subtypes, including H7N7, H7N9 and H15N8 (Figure 5c). Similarly, although 4B7 4D5 targeted a conserved epitope in the stalk, this antibody only reacted with HA of the H7N7 and H7N9 subtypes (Figure 5c). These findings suggested that three antibodies targeting the stalk epitopes exhibited cross-reactivity with different subtypes and 5D3 1B5 had a broad cross-group reactivity.

\section{Discussion}

Numerous vaccine candidates were generated in response to the emergence of H7N9 avian influenza. Some types of H7N9 vaccines induce low $\mathrm{HI}$ and VN antibody titers but high levels of IgG antibody in humans 
and animals, indicating that the antibody immunity against H7N9 vaccines is characterized with the potent elicitation of nonNeu antibodies. However, the antigenic epitopes underpinning this unique antibody response are still undefined. In this study, four novel nonNeu epitopes in the HA protein of H7N9 virus were identified through mapping with a panel of HA-specific mAbs. More importantly, two immunodominant epitopes in HA were defined by competition ELISA between the mAbs with chicken nonNeu antisera. Interestingly, nonNeu antisera against NDV-H7N9 vaccine and inactivated H7N9 antigen may target different dominant epitopes. In addition, the stalk epitopes identified in the present study are conserved among the H1-H17 subtypes and the mAbs targeting these epitopes exhibited cross-reactivity with different subtypes.

Vaccines against the H7 subtype influenza, including those against the H7N9 subtype, tend to induce a robust nonNeu antibody response (Bart et al., 2014; Couch, Patel, Wade-Bowers, \& Nino, 2012; Kamal et al., 2017). Several clinical studies showed that different types of H7N9 vaccines elicited low HI and NV titers in adults and booster immunization or addition of adjuvants was required to improve immunogenicity (Bart et al., 2014; Jackson et al., 2015). Similar findings were also obtained from animal studies on H7N9 vaccines (Hu, Liu, Jiao, \& Liu, 2017; Kamal et al., 2017; Liu Q. et al., 2015). It is noted that nonNeu IgG antibodies elicited by H7N9 vaccines and nonNeu H7N9-specific mAbs provide potent protection through Fc-mediated immune effector functions (Dunand et al., 2016; Stadlbauer et al., 2017; Tan et al., 2016). This unique immunophenotype indicates that the antigenic epitopes underlying the induction of nonNeu antibodies may be dominant in the HA protein of H7N9 virus. In this study, a panel of 15 HA-specific mAbs was generated and intriguingly, 14 of them had no HI and VN activities. Our team reported that NDV-vectored H7N9 vaccine elicited undetectable $\mathrm{HI} / \mathrm{VN}$ titers but high levels of nonNeu antibodies in chickens, which can serve as a good model to explore the molecular mechanisms for antibody immunity against H7N9 vaccine (Shi et al., 2019) Herein, nonNeu sera were also prepared in mice immunized with inactivated H7N9 antigen. Therefore, the availability of these materials provides an opportunity to define the nonNeu epitopes and screen the immunodominant epitopes in H7N9 HA.

Four epitopes, including one in the head and three in the stalk, targeted by nonNeu mAbs were identified using peptide-based ELISA and/or panning of phage display peptide library. Two mAbs, 4B7 4D5 and 1B10 1D1, exhibited a robust inhibitory effect on the nonNeu NDV-H7N9 sera, rather than on the neutralizing rGD15 sera, with a greater inhibition observed for the former. The epitopes recognized by these two mAbs were defined as the immunodominant epitopes in the context of NDV-H7N9 immunization in chickens, supporting the dominance of nonNeu epitopes in this vaccine model. Antibodies elicited by the non-adjuvanted inactivated H7N9 antigen in mice also had no virus neutralizing activity, which displayed a similar reactivity pattern with the potential linear B cell epitopes in HA compared to the NDV-H7N9 sera. Interestingly, 4B7 4D5 showed a low inhibition against murine nonNeu antisera, suggesting that nonNeu antibodies induced by whole-virus inactivated H7N9 antigen may target different dominant epitopes in HA. This phenomenon may be associated with different conformations of the HA protein in inactivated and NDV-vectored H7N9 vaccines (Hu et al., 2020). Moreover, the HA protein expressed in NDV backbone may be involved in replication of vaccine virus, which implicates conformational changes of HA and exposure of more antigenic epitopes.

The epitope recognized by 4B7 4D5 located in the stalk and showed a more potent inhibitory impact, indicating an important role of the stalk in the antibody response to NDV-H7N9 vaccine. H7N9 immunization in humans induces high levels of cross-reactive stalk-specific antibody immunity, which may be mainly resulted from the pre-existing anti-stalk immunity (Guo et al., 2014; Liu et al., 2017). Recently, the induction of broadly-reactive stalk-specific antibodies by H7N9 vaccines was detected in naïve chickens, suggesting that this antibody response profile may be an immunologic nature for H7N9 virus (Hu et al., 2020). Herein, the 4B7 4D5 epitope located in the stalk was identified as immunodominant, further strengthening the role of the stalk in the antibody response to viral-vectored H7N9 vaccines. However, a low competition was detected between 4B7 4D5 and mouse nonNeu sera, indicating that the HA stalk may be not dominant in immunization with non-adjuvanted inactivated H7N9 antigen. Consistently, Kamal et al. also demonstrated that immunization with whole inactivated $\mathrm{H} 7$ vaccines in mice elicited high levels of nonNeu antibodies, which predominantly directed against the globular head of HA, rather than the more conserved stalk region (Kamal et al., 2017). Therefore, the predominant epitopes underlying the elicitation of nonNeu antibodies 
by inactivated H7N9 vaccine remain to be determined.

Particularly, a linear motif "373-TAA-375" in the stalk was identified as a nonNeu epitope. This motif is also a part of a defined neutralizing epitope recognized by the CR $8020 \mathrm{mAb}$ with a cross-neutralizing activity against most group 2 influenza viruses (Ekiert et al., 2011). Moreover, a recent study reported that four H7N9 mAbs with cross-neutralizing activity against group 1 and 2 viruses target a liner epitope in the A helix domain of stalk containing the "TAA" motif (Li et al., 2019). However, whether the "TAA" motif is the core sequence of these epitopes and its contribution to virus neutralizing activity were not determined. The "TAA" motif is the key component of the N-cap domain in the stalk, which is thought to make a substantial energetic contribution to the membrane fusion process (Chen, Skehel, \& Wiley, 1999). This motif was identified as a nonNeu epitope in the present study, suggesting its minor role in the induction of neutralizing antibodies.

Current knowledge about the epitopes in H7N9 HA is mainly gained from studies on bioinformatic analysis (Liu, Song, Hua, Wu, \& Jiang, 2015; Wang et al., 2016), characterization of escape mutants (Sun et al., 2018; Thornburg et al., 2016) and crystal structural analysis (Dunand et al., 2016; Huang et al., 2019) based on neutralizing mAbs. There is still a lack of understanding of the nonNeu epitopes and immunodominant epitopes, especially in the polyclonal antibody response against H7N9 vaccines. Recently, Yao et al. identified a linear epitope 103-RESGG-107 recognized by three nonNeu H7N9-specific mAbs and reported high competitions between these antibodies and H7-positive sera, indicating that this epitope may be dominant in H7 immunization (Yao et al., 2020). Nevertheless, in their study, only mAbs against the HA globular head were investigated and the HA1 protein was used as the antigen in competition assay. Therefore, their findings cannot reflect the role of the epitopes in the stalk. In our study, the mAbs against the whole HA ectodomain were analyzed and thus identification of the nonNeu epitopes and dominant epitopes in HA in this study would help to fill the gaps for understanding the immunogenicity of H7N9 virus.

Another significance of our results is to provide implications for vaccine optimization. Low HI titers induced by H7N9 vaccines may limit large-scale evaluation of H7N9 vaccine efficacy in humans and animals because more expensive, labor- and time-consuming serological assays, such as ELISA, must be performed. Based on our findings, it is viable to skew the antibody immunity towards the HI or VN epitopes through modifying the dominant nonNeu epitopes, such as hyperglycosylation, which is known as the "immune redirecting" strategy used for many viral vaccines (Bajic et al., 2019; Bernstein et al., 2020; Ringe et al., 2019).

In summary, four novel nonNeu epitopes in the HA protein of H7N9 virus and two immunodominant epitopes in the process of immunization with viral-vectored H7N9 vaccine were defined. In addition, nonNeu antibodies induced by inactivated and viral-vectored H7N9 vaccines target different dominant epitopes in HA. Our study presented new data for understanding the molecular basis of antibody immunity against H7N9 vaccines and important information for vaccine design.

\section{ACKNOWLEDGEMENTS}

This work was supported by the National Key Research and Development Project of China (2016YFD0501601), the Open Project Program of Jiangsu Key Laboratory of Zoonosis (No. R1808), the Earmarked Fund for China Agriculture Research System (No.CARS-40), the 'Qing Lan Project' of Higher Education Institutions of Jiangsu Province, China, the 'High-end Talent Support Program' of Yangzhou University, China, and by A Project Funded by the Priority Academic Program Development of Jiangsu Higher Education Institutions (PAPD).

\section{CONFLICT OF INTEREST}

There are no conflicts of interest.

\section{ETHICAL APPROVAL}

The authors confirm that the ethical policies of the journal, as noted on the journal's author guidelines page, have been adhered to. All animal experiments were approved by the Jiangsu Administrative Committee 
for Laboratory Animals (Permission number: SYXK-SU-2007-0005), and complied with the guidelines of Jiangsu laboratory animal welfare and ethics of Jiangsu Administrative Committee of Laboratory Animals. Experiments involving live H7N9 viruses were performed in animal biosafety level-3 facilities, which were approved by the Ministry of Agriculture and Rural Affairs of the People's Republic of China.

\section{DATA AVAIL ABILIT Y S TATEMENT}

The data that support the findings of this study are available from the corresponding author upon reasonable request.

\section{REFERENCES}

Bajic, G., Maron, M. J., Adachi, Y., Onodera, T., McCarthy, K. R., McGee, C. E., . . . Schmidt, A. G. (2019). Influenza Antigen Engineering Focuses Immune Responses to a Subdominant but Broadly Protective Viral Epitope. Cell host $\mathcal{E}$ microbe, 25 (6), 827-835 e826. doi:10.1016/j.chom.2019.04.003

Bart, S. A., Hohenboken, M., Della Cioppa, G., Narasimhan, V., Dormitzer, P. R., \& Kanesa-Thasan, N. (2014). A cell culture-derived MF59-adjuvanted pandemic A/H7N9 vaccine is immunogenic in adults. Science translational medicine, 6 (234), 234ra255. doi:10.1126/scitranslmed.3008761

Bernstein, D. I., Guptill, J., Naficy, A., Nachbagauer, R., Berlanda-Scorza, F., Feser, J., . . Krammer, F. (2020). Immunogenicity of chimeric haemagglutinin-based, universal influenza virus vaccine candidates: interim results of a randomised, placebo-controlled, phase 1 clinical trial. The Lancet. Infectious diseases, 20 (1), 80-91. doi:10.1016/S1473-3099(19)30393-7

Chen, J., Skehel, J. J., \& Wiley, D. C. (1999). N- and C-terminal residues combine in the fusion-pH influenza hemagglutinin $\mathrm{HA}(2)$ subunit to form an $\mathrm{N}$ cap that terminates the triple-stranded coiled coil. Proceedings of the National Academy of Sciences of the United States of America, 96 (16), 8967-8972. doi:10.1073/pnas.96.16.8967

Couch, R. B., Patel, S. M., Wade-Bowers, C. L., \& Nino, D. (2012). A randomized clinical trial of an inactivated avian influenza A (H7N7) vaccine. PloS one, 7 (12), e49704. doi:10.1371/journal.pone.0049704

Dunand, C. J. H., Leon, P. E., Huang, M., Choi, A., Chromikova, V., Ho, I. Y., . . . Wilson, P. C. (2016). Both Neutralizing and Non-Neutralizing Human H7N9 Influenza Vaccine-Induced Monoclonal Antibodies Confer Protection. Cell host $\& 3$ microbe, 19 (6), 800-813. doi:10.1016/j.chom.2016.05.014

Ekiert, D. C., Friesen, R. H., Bhabha, G., Kwaks, T., Jongeneelen, M., Yu, W., . . . Goudsmit, J. (2011). A highly conserved neutralizing epitope on group 2 influenza A viruses.Science, 333 (6044), 843-850. doi:10.1126/science.1204839

Gao, R., Cao, B., Hu, Y., Feng, Z., Wang, D., Hu, W., . . . Shu, Y. (2013). Human infection with a novel avian-origin influenza A (H7N9) virus. The New England journal of medicine, 368 (20), 1888-1897. doi:10.1056/NEJMoa1304459

Guo, L., Zhang, X., Ren, L., Yu, X., Chen, L., Zhou, H., . . . Wang, J. (2014). Human antibody responses to avian influenza A(H7N9) virus, 2013. Emerging infectious diseases, 20 (2), 192-200. doi:10.3201/eid2002.131094

Hu, Z., Liu, X., Jiao, X., \& Liu, X. (2017). Newcastle disease virus (NDV) recombinant expressing the hemagglutinin of H7N9 avian influenza virus protects chickens against NDV and highly pathogenic avian influenza A (H7N9) virus challenges. Vaccine, 35 (48), 6585-6590. doi:10.1016/j.vaccine.2017.10.010

Hu, Z., Shi, L., Xu, N., Wang, X., Hu, J., Zhao, J., . . Cao, Y. (2020). Induction of cross-group broadly reactive antibody response by natural H7N9 avian influenza virus infection and immunization with inactivated H7N9 vaccine in chickens. Transboundary and emerging diseases . doi:10.1111/tbed.13705

Hu, Z., Zhao, J., Zhao, Y., Fan, X., Hu, J., Shi, L., . . . Liu, X. (2019). Hemagglutinin-Specific Nonneutralizing Antibody Is Essential for Protection Provided by Inactivated and Viral-Vectored H7N9 Avian 
Influenza Vaccines in Chickens. Frontiers in veterinary science, 6 , 482. doi:10.3389/fvets.2019.00482

Huang, K. A., Rijal, P., Jiang, H., Wang, B., Schimanski, L., Dong, T., . . . Townsend, A. R. (2019). Structure-function analysis of neutralizing antibodies to H7N9 influenza from naturally infected humans. Nature microbiology, 4 (2), 306-315. doi:10.1038/s41564-018-0303-7

Isakova-Sivak, I., \& Rudenko, L. (2017). Tackling a novel lethal virus: a focus on H7N9 vaccine development. Expert review of vaccines, 16 (7), 1-13. doi:10.1080/14760584.2017.1333907

Jackson, L. A., Campbell, J. D., Frey, S. E., Edwards, K. M., Keitel, W. A., Kotloff, K. L., . . . Bellamy, A. R. (2015). Effect of Varying Doses of a Monovalent H7N9 Influenza Vaccine With and Without AS03 and MF59 Adjuvants on Immune Response: A Randomized Clinical Trial. Jama, 314 (3), 237-246. doi:10.1001/jama.2015.7916

Jiang, W., Hou, G., Li, J., Peng, C., Wang, S., Liu, S., . . Liu, H. (2019). Prevalence of H7N9 subtype avian influenza viruses in poultry in China, 2013-2018. Transboundary and emerging diseases, 66 (4), 1758-1761. doi:10.1111/tbed.13183

Kamal, R. P., Blanchfield, K., Belser, J. A., Music, N., Tzeng, W. P., Holiday, C., . . York, I. A. (2017). Inactivated $\mathrm{H} 7$ influenza virus vaccines protect mice despite low levels of neutralizing antibodies. $J$ Virol . doi:10.1128/JVI.01202-17

Krammer, F., \& Cox, R. J. (2013). The emergence of H7N9 viruses: a chance to redefine correlates of protection for influenza virus vaccines. Expert review of vaccines, 12 (12), 1369-1372. doi:10.1586/14760584.2013.850036

Li, Z., Wan, Z., Li, T., Xie, Q., Sun, H., Chen, H., . . Ye, J. (2019). A novel linear epitope crossing Group 1 and Group 2 influenza A viruses located in the helix A of HA2 derived from H7N9. Veterinary microbiology, 228 , 39-44. doi:10.1016/j.vetmic.2018.11.002

Liu, D., Zhang, Z., He, L., Gao, Z., Li, J., Gu, M., . . . Liu, X. (2018). Characteristics of the emerging chicken-origin highly pathogenic H7N9 viruses: A new threat to public health and poultry industry. Journal of Infection, 76 (2), 217-220. doi:10.1016/j.jinf.2017.09.005

Liu, L., Nachbagauer, R., Zhu, L., Huang, Y., Xie, X., Jin, S., . . Xu, J. (2017). Induction of Broadly Cross-Reactive Stalk-Specific Antibody Responses to Influenza Group 1 and Group 2 Hemagglutinins by Natural H7N9 Virus Infection in Humans. The Journal of infectious diseases, 215 (4), 518-528. doi:10.1093/infdis/jiw608

Liu, M., Song, T., Hua, S., Wu, A., \& Jiang, T. (2015). Computational analysis of antigenic epitopes of avian influenza A (H7N9) viruses. Science China. Life sciences, 58 (7), 687-693. doi:10.1007/s11427-015-4886-4

Liu, Q., Mena, I., Ma, J., Bawa, B., Krammer, F., Lyoo, Y. S., . . Richt, J. A. (2015). Newcastle Disease Virus-Vectored H7 and H5 Live Vaccines Protect Chickens from Challenge with H7N9 or H5N1 Avian Influenza Viruses. J Virol, 89 (14), 7401-7408. doi:10.1128/JVI.00031-15

Ma, M., Yang, Y., \& Fang, L. (2018). Highly Pathogenic Avian H7N9 Influenza Viruses: Recent Challenges.Trends in microbiology . doi:10.1016/j.tim.2018.11.008

Ringe, R. P., Pugach, P., Cottrell, C. A., LaBranche, C. C., Seabright, G. E., Ketas, T. J., . . Moore, J. P. (2019). Closing and Opening Holes in the Glycan Shield of HIV-1 Envelope Glycoprotein SOSIP Trimers Can Redirect the Neutralizing Antibody Response to the Newly Unmasked Epitopes. Journal of virology, 93 (4). doi:10.1128/JVI.01656-18

Shi, J., Deng, G., Ma, S., Zeng, X., Yin, X., Li, M., . . . Chen, H. (2018). Rapid Evolution of H7N9 Highly Pathogenic Viruses that Emerged in China in 2017. Cell host 83 microbe, 24 (4), 558-568 e557. doi:10.1016/j.chom.2018.08.006 
Shi, L., Hu, Z., Hu, J., Liu, D., He, L., Liu, J., . . Liu, X. (2019). Single Immunization with Newcastle Disease Virus-Vectored H7N9 Vaccine Confers a Complete Protection Against Challenge with Highly Pathogenic Avian Influenza H7N9 Virus.Avian diseases, 63 (1), 61-67. doi:10.1637/11965-090118-Reg.1

Stadlbauer, D., Rajabhathor, A., Amanat, F., Kaplan, D., Masud, A., Treanor, J. J., . . Krammer, F. (2017). Vaccination with a Recombinant H7 Hemagglutinin-Based Influenza Virus Vaccine Induces Broadly Reactive Antibodies in Humans.mSphere, 2 (6). doi:10.1128/mSphere.00502-17

Sun, Z., Shi, B., Meng, F., Ma, R., Hu, Q., Qin, T., . . Liu, X. (2018). Development of a Colloidal Gold-Based Immunochromatographic Strip for Rapid Detection of H7N9 Influenza Viruses. Frontiers in microbiology, 9 , 2069. doi:10.3389/fmicb.2018.02069

Tan, G. S., Leon, P. E., Albrecht, R. A., Margine, I., Hirsh, A., Bahl, J., \& Krammer, F. (2016). Broadly-Reactive Neutralizing and Non-neutralizing Antibodies Directed against the H7 Influenza Virus Hemagglutinin Reveal Divergent Mechanisms of Protection. PLoS pathogens, 12 (4), e1005578. doi:10.1371/journal.ppat.1005578

Thornburg, N. J., Zhang, H., Bangaru, S., Sapparapu, G., Kose, N., Lampley, R. M., . . Crowe, J. E., Jr. (2016). H7N9 influenza virus neutralizing antibodies that possess few somatic mutations. The Journal of clinical investigation, 126 (4), 1482-1494. doi:10.1172/JCI85317

Wang, X., Jiang, H., Wu, P., Uyeki, T. M., Feng, L., Lai, S., . . Yu, H. (2017). Epidemiology of avian influenza A H7N9 virus in human beings across five epidemics in mainland China, 2013-17: an epidemiological study of laboratory-confirmed case series. The Lancet. Infectious diseases, 17 (8), 822-832. doi:10.1016/S14733099(17)30323-7

Wang, X., Sun, Q., Ye, Z., Hua, Y., Shao, N., Du, Y., . . . Wan, C. (2016). Computational approach for predicting the conserved B-cell epitopes of hemagglutinin $\mathrm{H} 7$ subtype influenza virus. Experimental and therapeutic medicine, 12 (4), 2439-2446. doi:10.3892/etm.2016.3636

Wong, S. S., Jeevan, T., Kercher, L., Yoon, S. W., Petkova, A. M., Crumpton, J. C., . . Webby, R. J. (2014). A single dose of whole inactivated H7N9 influenza vaccine confers protection from severe disease but not infection in ferrets. Vaccine, 32 (35), 4571-4577. doi:10.1016/j.vaccine.2014.06.016

Yang, F., Xiao, Y., Lu, R., Chen, B., Liu, F., Wang, L., . . Wu, H. (2020). Generation of neutralizing and non-neutralizing monoclonal antibodies against H7N9 influenza virus. Emerging microbes 83 infections, 9 (1), 664-675. doi:10.1080/22221751.2020.1742076

Yao, L., Chen, Y., Wang, X., Bi, Z., Xiao, Q., Lei, J., . . Yan, L. (2020). Identification of antigenic epitopes in the haemagglutinin protein of $\mathrm{H} 7$ avian influenza virus.Avian pathology : journal of the W.V.P.A, 49 (1), 62-73. doi:10.1080/03079457.2019.1666971

Zhang, F., Bi, Y., Wang, J., Wong, G., Shi, W., Hu, F., . . Gao, G. F. (2017). Human infections with recently-emerging highly pathogenic H7N9 avian influenza virus in China. The Journal of infection, 75 (1), 71-75. doi:10.1016/j.jinf.2017.04.001

\section{Figure legends}

FIGURE 1 Expression of the HA protein in baculovirus expression system. (a) Detection of HA expression in sf9 cells inoculated with the recombinant baculovirus using indirect immunofluorescence assay. Scale bar, $200 \mu \mathrm{m}$. (b) SDS-PAGE analysis of the purified HA protein. M, marker; lane 1, elution with $30 \mathrm{mM}$ imidazole; lane 2, elution with $60 \mathrm{mM}$ imidazole; lane 3, elution with $250 \mathrm{mM}$ imidazole. (c) Detection of HA expression using Western blot under non-reducing conditions.

FIGURE 2 Epitope mapping of the mAbs using peptide-based ELISA. Reactivity of 1A1 1D7 (a), 1A12 2A4 (b), 2E3 3D8 (c) and 5D3 1B5 (d) with 19 potential linear B cell epitopes in HA was determined using peptide-based ELISA. The HA protein and bovine serum albumin (BSA) was used as the positive and negative control respectively. (e) The overlap motif between peptide 12 and 13 was truncated into three 
overlapping short peptides. (f) The peptide 19 was truncated into five overlapping short peptides. Reactivity of 1A1 1D7 (g), 1A12 2A4 (h), 2E3 1D8 (i) and 5D3 1B5 (j) with the truncated peptides was determined using peptide-ELISA. The BSA was used as the negative control.

FIGURE 3 Reactivity of the mAbs with H7N9 virus determined by IFA. MDCK cells were infected with H7N9 GD15 strain at a MOI of 0.1. After $30 \mathrm{~h}$, the cells were fixed and incubated with antiH7N9 mAbs (1:500) as the primary antibodies. Goat anti-mouse IgG Alexa 488 was used as the secondary antibody. Non-infected cells that were treated in a same manner were used as the mock control. Scale bar, $200 \mu \mathrm{m}$.

FIGURE 4 Identification of the immunodominant epitopes in HA using competition ELISA. (a) Reactivity of chicken NDV-H7N9 immune sera with potential B cell epitopes. (b) Reactivity of mouse anti-H7N9 sera with potential B cell epitopes. (c) Competition between non-neutralizing mAbs and chicken NDV-H7N9 immune sera. (d) Competition between the mAbs (4B7 4D5 and 1B10 1D1) and chicken NDVH7N9 sera. (e) Competition of 4B7 4D5 and 1B10 1D1 with chicken rGD15 immune sera. (f) Competition between 4B7 4D5 and mouse anti-H7N9 sera. Adj, adjuvanted; Non-adj, non-adjuvanted. (g, h, i) Area under curve (AUC) was calculated based on the curves of percentage of inhibition (PI) and PI AUC values were analyzed using one-way ANOVA. ${ }^{*}, p<0.05 ; * *, p<0.01$; ns, non-significant.

FIGURE 5 Conformational analysis of the epitopes in HA and cross-reactivity of the stalkreactive mAbs. (a) Localization of the identified epitopes were illustrated in the HA monomer. (b) Sequence variation analysis of the epitopes recognized by the mAbs. The HA sequences of H1-H17 subtypes were aligned using BioEdit software and variation of the epitopes was illustrated using Weblogo. The size of letters correlates with the conservation of the amino acid. (c) Reactivity of the mAbs with different subtypes. The HA proteins belonging to group 1 (H1N1, H5N1 and H9N2) and group 2 (H7N7, H7N9 and H15N8) were used. The bovine serum albumin (BSA) was used as the negative control.
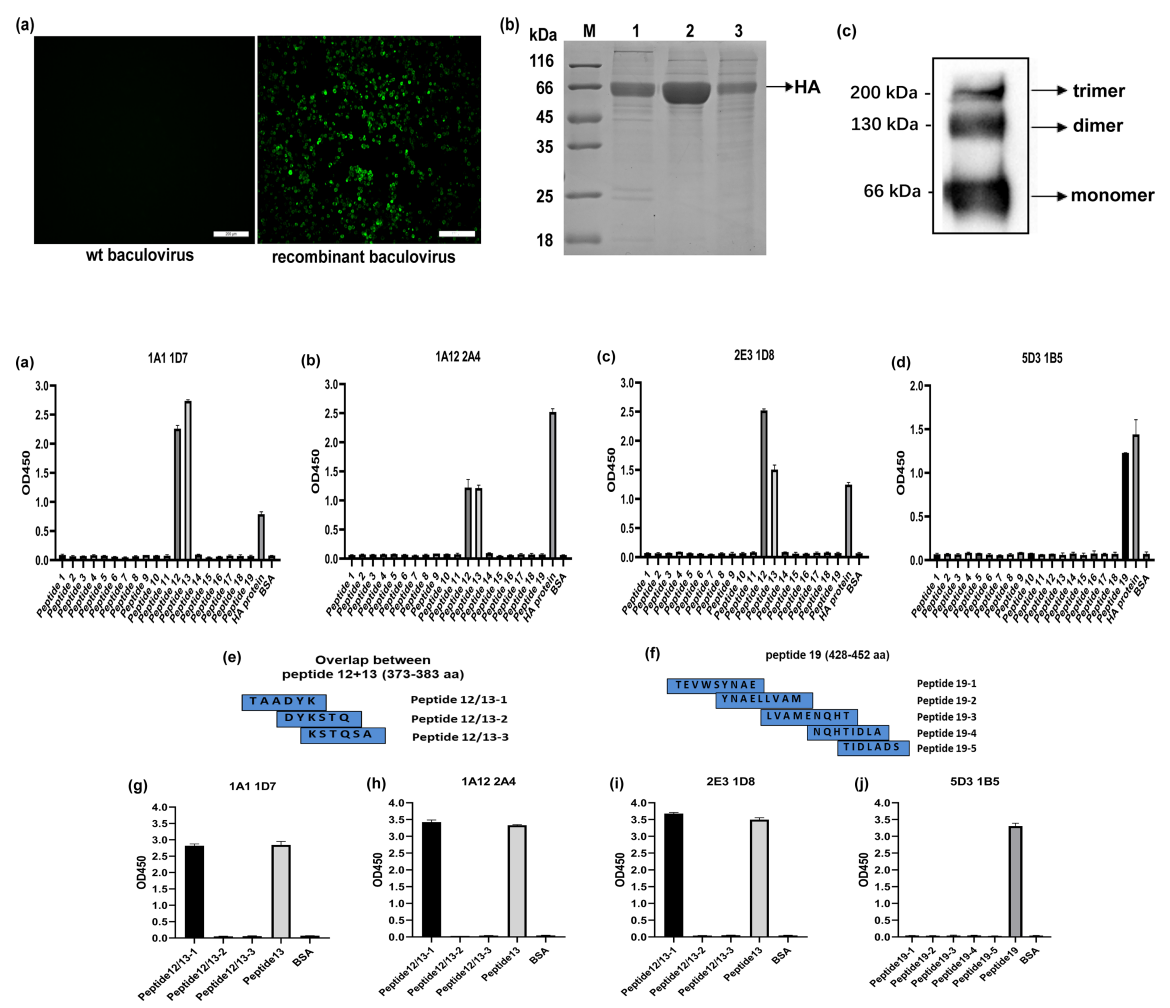

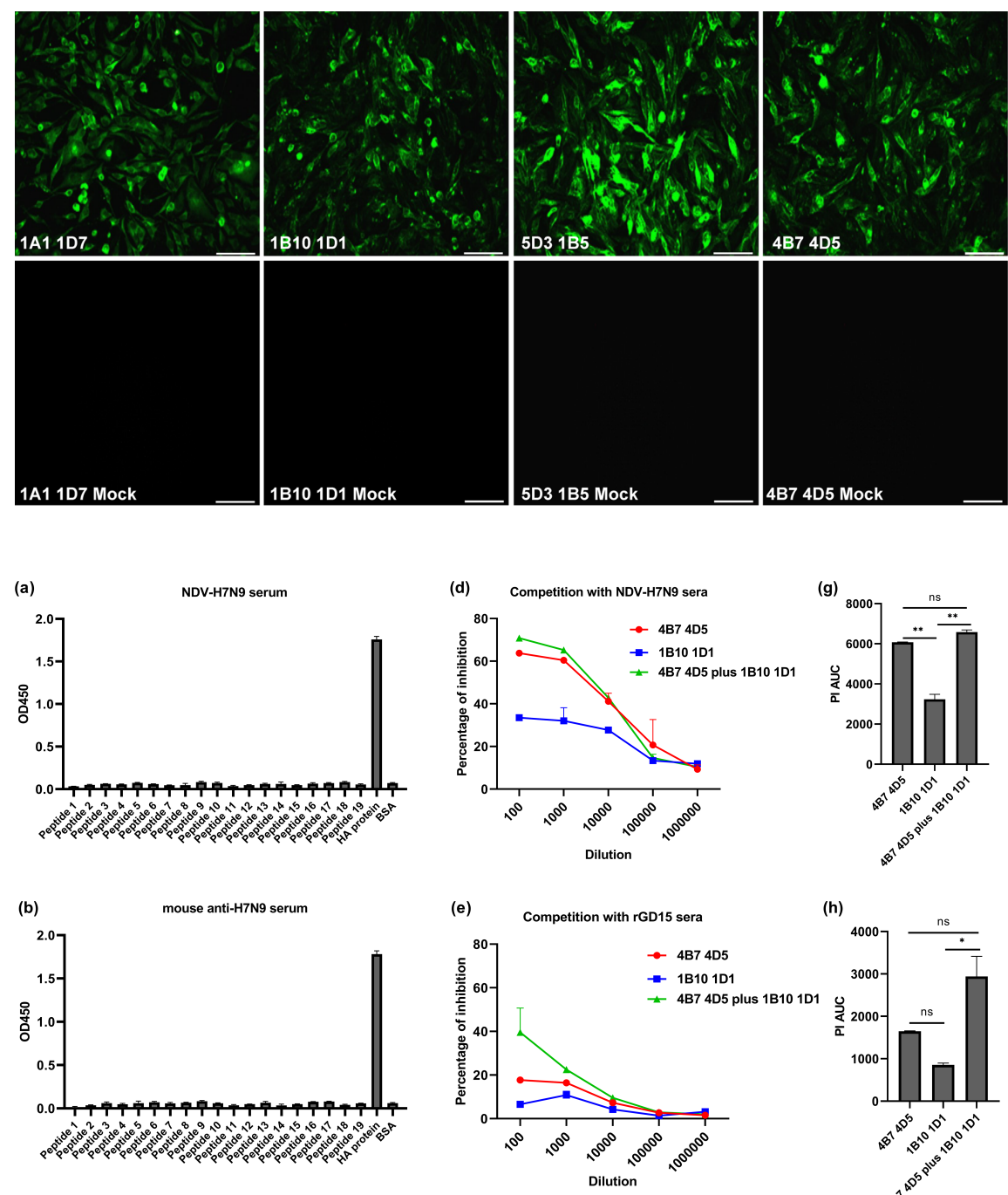

(h)
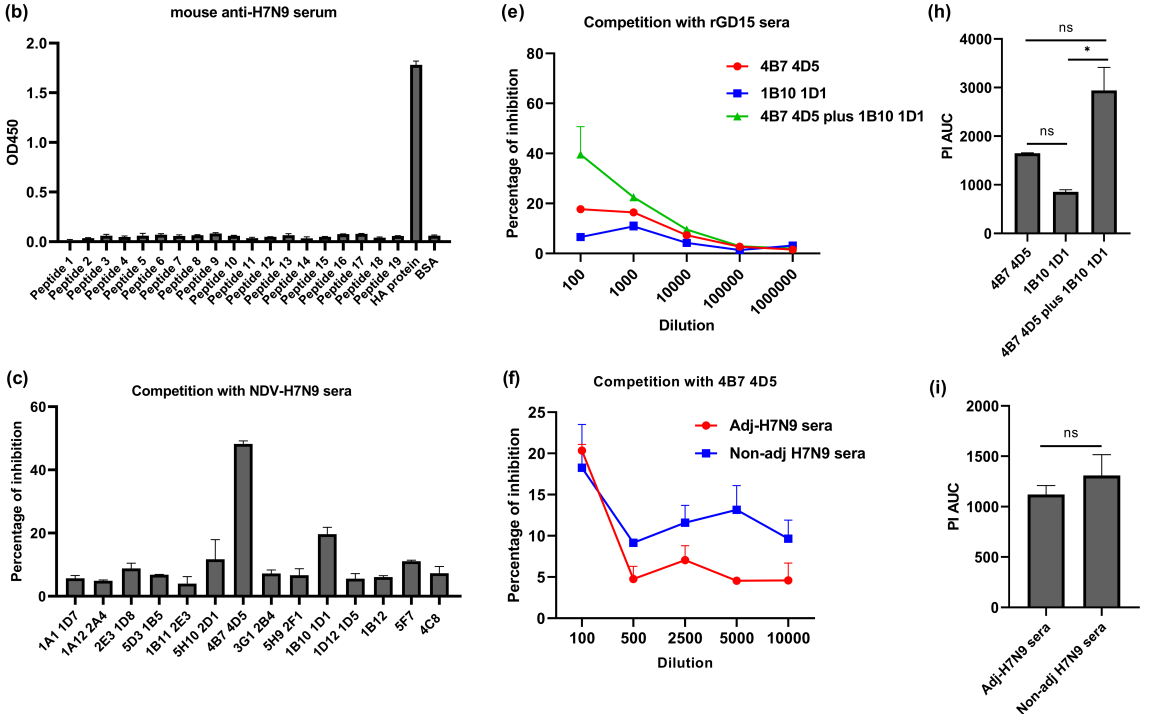


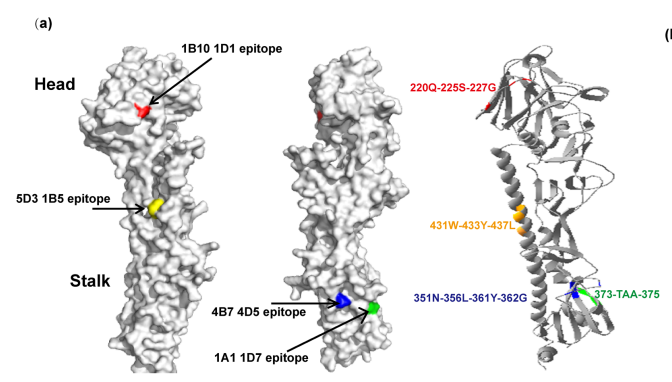

(b)

(c)
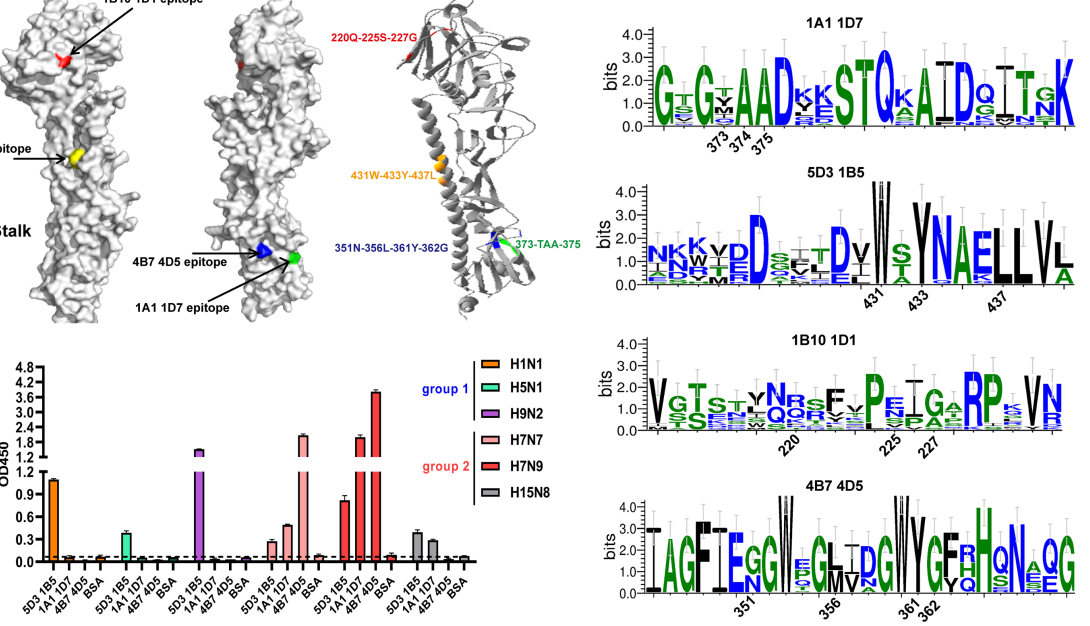

4.0青 $1 \mathrm{~B} 101 \mathrm{D} 1$
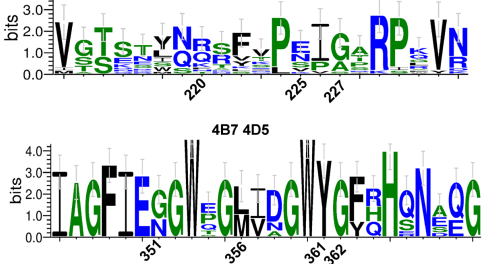

\section{Hosted file}

Table 1.pdf available at https://authorea.com/users/364101/articles/484641-identificationof-the-dominant-non-neutralizing-epitopes-in-the-haemagglutinin-of-h7n9-avian-influenzavirus

\section{Hosted file}

Table 2.pdf available at https://authorea.com/users/364101/articles/484641-identificationof-the-dominant-non-neutralizing-epitopes-in-the-haemagglutinin-of-h7n9-avian-influenzavirus

\section{Hosted file}

Table 3.pdf available at https://authorea.com/users/364101/articles/484641-identificationof-the-dominant-non-neutralizing-epitopes-in-the-haemagglutinin-of-h7n9-avian-influenzavirus 